\title{
Syntenin increases the invasiveness of small cell lung cancer cells by activating p38, AKT, focal adhesion kinase and SP1
}

\author{
Wook Youn Kim ${ }^{1,2,3}$, Ji-young Jang ${ }^{2}$, Yoon Kyung Jeon ${ }^{1,2}$, Doo Hyun Chung ${ }^{1}$, Young Goo Kim ${ }^{2}$ \\ and Chul-Woo Kim ${ }^{1,2}$
}

Syntenin is a PDZ domain-containing adaptor protein that has been recently shown to regulate migration and invasion in several tumors. Small cell lung cancer (SCLC) is notorious for its invasiveness and strong potential for metastasis. We therefore studied the influence of syntenin on the invasiveness of SCLC. Immunohistochemistry in tumor tissues showed that syntenin was more frequently expressed in small cell carcinomas than other neuroendocrine tumors, such as carcinoids and neuroblastomas, suggesting that syntenin expression may be related to more aggressive forms of neuroendocrine tumors. In SCLC patients, syntenin overexpression in tumor cells was significantly associated with more extensive and advanced disease at the time of diagnosis $(P=0.029)$. Overexpression of syntenin in SCLC cells that were intrinsically syntenin-low increased the invasiveness of cells and led to the induction of extracellular matrix (ECM)-degrading membrane type 1-matrix metalloproteinase (MT1MMP) and matrix metalloproteinase 2 (MMP2). In contrast, suppression of syntenin in syntenin-high cells was associated with the downregulation of MT1-MMP. Contrary to the results of previous studies using malignant melanomas and breast carcinomas, signaling cascades were shown to be further transduced through p38 MAPK and PI3K/AKT, with activation of SP1 rather than $\mathrm{NF}-\mathrm{\kappa B}$, under circumstances not involving ECM interaction. In addition, the upstream molecule focal adhesion kinase was induced by syntenin activation, in spite of the absence of ECM interaction. These results suggest that syntenin might contribute to the invasiveness of SCLC and could be utilized as a new therapeutic target for controlling invasion and metastasis in SCLC. Experimental \& Molecular Medicine (2014) 46, e90; doi:10.1038/emm.2014.1; published online 11 April 2014

Keywords: MMP; p38 MAPK; PI3K/AKT; small cell lung cancer; syntenin

\section{INTRODUCTION}

The major characteristics of malignant tumor cells are their ability to invade foreign tissues and form metastatic foci at distant locations in the body. Such processes require tumor cell attachment to various matrix proteins and the degradation of the extracellular matrix (ECM), mainly by matrix metalloproteinase (MMP), followed by the migration of tumor cells into the surrounding stroma. Syntenin, also known as melanoma differentiation associated gene- 9 , is an adaptor protein containing the PDZ domain that is involved in the organization of protein complexes in the plasma membranes, regulation of B-cell development, intracellular trafficking and cell-surface targeting, synaptic transmission and axonal outgrowth. ${ }^{1-4}$ Recent studies have defined a seminal role for syntenin in cancer metastasis.
The invasiveness-associated role of syntenin was first observed in malignant melanoma. ${ }^{5-8}$ Results from recent studies using malignant melanoma cell lines have shown that syntenin might function as an important regulator, controlling the invasiveness and metastatic potential in malignant melanoma. ${ }^{9}$ In a matched set of melanoma cell lines of lowly and highly metastatic cells, syntenin expression was significantly higher in highly metastatic cells; overexpression of syntenin significantly accelerated migration and invasion, whereas forced inhibition of syntenin significantly suppressed invasiveness. $^{9}$

In another study using epithelial cancer cell lines, overexpression of syntenin caused a significant increase in invasion and migration properties in gastric and breast cancer cell lines, resulting in morphologic changes with increased pseudopodia

\footnotetext{
${ }^{1}$ Department of Pathology, Seoul National University College of Medicine, Seoul, Korea; ${ }^{2}$ The Tumor Immunity Medical Research Center, Cancer Research Center, Seoul National University College of Medicine, Seoul, Korea and ${ }^{3}$ Department of Pathology, Konkuk University School of Medicine, Seoul, Korea Correspondence: Dr C-W Kim, Department of Pathology, Seoul National University College of Medicine, 28 Yeongeon-dong, Jongro-gu, Seoul 110-799, Korea.

E-mail: cwkim@snu.ac.kr
}

Received 19 April 2013; revised 2 November 2013; accepted 16 December 2013 
formation. ${ }^{10}$ Similar results were found in a study using breast and bladder cancer cells; this study revealed that the synteninrelated effect could be regulated by the binding of ligand to the PDZ domains. ${ }^{11}$

How syntenin transduces the activated signal inside the cell has been studied in several cell lines. Characteristically, syntenin signaling was differentially activated, depending on the cell lines and ECM stimulants used. For example, in malignant melanoma cells stimulated with fibronectin, syntenin facilitated the phosphorylation of focal adhesion kinase (FAK) and activation of $\mathrm{c}$-SRC, followed by the activation of p38 MAPK and, subsequently, NF- $\kappa \mathrm{B}$, finally leading to the production of ECM-degrading MMPs. ${ }^{12,13}$ However, in breast cancer cell lines, interactions with type 1 collagen elicited syntenin activation, which led to PI3K/AKT and ERK pathway activation mediated by RhoA, cdc42, Racl and H-ras. ${ }^{11}$

Small cell lung cancer (SCLC) is notorious for having a strong potential for metastasis and invasion and has very poor prognosis, with a median survival of only 8 months and a 2-year survival rate of less than $5 \% .{ }^{14-16}$ Considering the contribution of syntenin to invasiveness of several cancers, we investigated the role of syntenin in SCLC.

In this study, we showed, for the first time in SCLC, that syntenin might promote the invasiveness and metastasis of SCLC via unique pathways, different from those in malignant melanoma or other carcinoma cells.

\section{MATERIALS AND METHODS}

\section{Cell lines and culture}

Six human SCLC lines, NCI-H69, NCI-H417, NCI-H187, NCI-H146, NCI-H209 and NCI-H889, were used as representative cell lines. All cell lines were purchased from the Korean Cell Line Bank (KCLB, Seoul, Korea). Cells were cultured in RPMI-1640 culture medium (Gibco, Grand Island, NY, USA), supplemented with 10\% (v/v) fetal calf serum or fetal bovine serum, 100 units $\mathrm{ml}^{-1}$ penicillin, and $100 \mu \mathrm{g} \mathrm{ml}^{-1}$ streptomycin (Gibco), in a humidified 5\% $\mathrm{CO}_{2} / 95 \%$ air atmosphere at $37^{\circ} \mathrm{C}$.

\section{Antibodies and reagents}

The following antibodies were used: rabbit polyclonal antibodies for AKT (Cell Signaling, Beverly, MA, USA), p38 (Santa Cruz Biotechnology, Santa Cruz, CA, USA), for p65 (Santa Cruz Biotechnology), p50 (Santa Cruz Biotechnology), phopho-c-jun (Cell Signaling), phospo-FAK (Invitrogen, Carlsbad, CA, USA) and IкB $\alpha$ (Santa Cruz Biotechnology); rabbit monoclonal antibodies for phospho-AKT (Cell Signaling) and c-jun (Cell Signaling); mouse monoclonal antibodies for syntenin (Abnova, Taipei, Taiwan) and FAK (BD Biosciences, San Jose, CA, USA).

\section{Syntenin expression vector and transfection}

An amino-terminally FLAG-tagged syntenin expression vector, generated by cloning syntenin cDNA into a pCMV-Tag 2 vector (Stratagene, La Jolla, CA, USA) within the frame, as previously described, ${ }^{10}$ was generously donated by Dr Jeong-Hyung Lee (Kangwon National University, Chuncheon, Korea). An empty pCMV-Tag2 vector, without syntenin cDNA, was used as negative control.
Cells were transfected with either the syntenin expression vector or the empty pCMV-Tag2 vector, using Lipofectamine 2000 (Invitrogen), in six-well plates $\left(8 \times 10^{5}\right.$ cells per well $)$ and cultured in serumstarved condition. Transfected cells were collected $12-24 \mathrm{~h}$ after transfection.

\section{Syntenin siRNA and transfection}

Syntenin siRNA was purchased from Santa Cruz Biotechnology (sc42164). Scrambled siRNA was purchased from Bioneer (Daejeon, Korea) and used as a negative control.

Cell lines were transfected with either syntenin siRNA or scrambled siRNA using Lipofectamine 2000 (Invitrogen) on six-well plates $\left(8 \times 10^{5}\right.$ cells per well). Transfected cells were then cultured for $6 \mathrm{~h}$, and culture media were replaced with fresh media supplemented with $10 \%$ fetal bovine serum. The cells were collected $48 \mathrm{~h}$ after transfection.

\section{Reverse transcription and quantitative real-time PCR}

After $24-48 \mathrm{~h}$ of transfection of either the syntenin expression vector or siRNA, the cells were collected and total RNA was extracted using Trizol (Invitrogen), according to the manufacturer's instructions. For RT-PCR analysis, $5 \mu \mathrm{g}$ total RNA was reverse-transcribed using RT-PCR kits (Promega, Madison, WI, USA). PCR was then used to amplify the target cDNA, using the following conditions: 35 cycles of $94{ }^{\circ} \mathrm{C}$ for $1 \mathrm{~min}, 55^{\circ} \mathrm{C}$ for $1 \mathrm{~min}$ and $72{ }^{\circ} \mathrm{C}$ for $2 \mathrm{~min}$. The PCR products were analyzed using standard agarose gel electrophoresis.

The primer sequences were as follows: Syntenin (forward primer, $5^{\prime}$-GTGGGCTAGAAGAATCCTGC- ${ }^{\prime}$ and reverse primer, $5^{\prime}$-ACTGT TCTGGGCTAGTGTAC-3'), MT1-MMP (forward primer, 5'-CCATT GGGCATCCAGAAGAGAGC- $3^{\prime}$ and reverse primer, $5^{\prime}$-GGATACCCA ATGCCCATTGGCCA-3'), MMP9 (forward primer, $5^{\prime}$-TTCATCTTC CAAGGCCAATC- $3^{\prime}$ and reverse primer, $5^{\prime}$-CTTGTCGCTGTCAAAG TTCG- $3^{\prime}$ ), MMP2 (forward primer, $5^{\prime}$-GGCTGGTCAGTGGCTTGG GGTA- $3^{\prime}$ and reverse primer, $5^{\prime}$-AGATCTTCTTCTTCAAGGACCGG TT-3'), VEGF (forward primer, $5^{\prime}$-CTACCTCCACCATGCCAAGT- $3^{\prime}$ and reverse primer, $5^{\prime}$-GCAGTAGCTGCGCTGCGCTGATAGA-3') and $\beta$-actin (forward primer, $5^{\prime}$-GGAAATCGTGCGTGACATTAA GG-3' and reverse primer, $5^{\prime}$-GGCTTTTAGGATGGCAAGGGAC- $3^{\prime}$ ).

For quantitative real-time PCR (qPCR), target cDNA was amplified and detected using the iCyler IQ real-time PCR system (Bio-Rad, Hercules, CA, USA) and SYBR-Green PCR Master Mix (TaKaRa, Otsu, Japan), according to the manufacturer's instructions. The qPCR conditions were as follows: $95^{\circ} \mathrm{C}$ for $3 \mathrm{~min},\left(95^{\circ} \mathrm{C}\right.$ for $30 \mathrm{~s}, 60^{\circ} \mathrm{C}$ for $45 \mathrm{~s})$ ( 40 cycles), $95^{\circ} \mathrm{C}$ for $60 \mathrm{~s}$, and $55^{\circ} \mathrm{C}$ for $60 \mathrm{~s}$.

All experiments were repeated three times, and the mRNA levels of each gene were normalized to those of GAPDH as an internal control. The primer sequences for qPCR were as follows: Syntenin (forward primer, $5^{\prime}$-TTCTGCTCCTATCCCTCACG-3' and reverse primer $5^{\prime}$-CCAGTTACAGGAGCCACCAT- $3^{\prime}$ ), MT1-MMP (forward primer, $5^{\prime}$-CCATTGGGCATCCAGAAGAGAGC- $3^{\prime}$ and reverse primer, $5^{\prime}$-GG ATACCCAATGCCCATTGGCCA-3'), MMP2 (forward primer, $5^{\prime}$-GG CTGGTCAGTGGCTTGGGGTA- $3^{\prime}$ and reverse primer, $5^{\prime}$-AGATCTT CTTCTTCAAGGACCGGTT- $3^{\prime}$ ) and GAPDH (forward primer, $5^{\prime}$-AC CACAGTCCATGCCATCAC- $3^{\prime}$ and reverse primer, $5^{\prime}$-TCCACCACC CTGTTGCTGTA- $3^{\prime}$ ).

\section{Western blot}

Whole cell lysates and either nuclear or cytoplasmic extracts were used. The cells were washed with ice-cold PBS (calcium- and magnesium-free phosphate buffered saline) two times and harvested 
by centrifugation at $15000 \times g$ for $5 \mathrm{~min}$ at $4{ }^{\circ} \mathrm{C}$. The whole cell lysates were extracted from the cell pellet using $0.1 \mathrm{ml}$ ice-cold lysis buffer $\left(5 \mathrm{mml}^{-1}\right.$ ethylenediamine tetra-acetic acid; $300 \mathrm{mml}^{-1} \mathrm{NaCl} ; 0.1 \%$ NP-40; $0.5 \mathrm{mml}^{-1} \mathrm{NaF} ; 0.5 \mathrm{mml}^{-1} \mathrm{Na}_{3} \mathrm{VO}_{4} ; 0.5 \mathrm{mml}^{-1}$ phenylmethylsulfonyl fluoride; and $10 \mu \mathrm{g} \mathrm{ml}^{-1}$ each of aprotinin, pepstatin and leupeptin; Sigma, St Louis, MO, USA).

To obtain cytoplasmic extracts, the harvested cell pellets were resuspended in $5 \mathrm{ml}$ of ice-cold hypotonic buffer (that is, $20 \mathrm{mml}^{-1}$ HEPES; $10 \mathrm{mMl}^{-1} \mathrm{KCl}$; $10 \%$ glycerol; $1 \mathrm{mml}^{-1}$ ethylenediamine tetraacetic acid; $0.5 \mathrm{mml}^{-1} \mathrm{NaF}$; $0.5 \mathrm{mml}^{-1} \mathrm{Na}_{3} \mathrm{VO}_{4}$; $0.5 \mathrm{mml}^{-1}$ phenylmethylsulfonyl fluoride; and $10 \mu \mathrm{g} \mathrm{ml}^{-1}$ each of aprotinin, pepstatin and leupeptin; Sigma), kept on ice for 5 min with tapping, and centrifuged at $15000 \times g$ for $1 \mathrm{~min}$ at $4{ }^{\circ} \mathrm{C}$. The supernatant contained the cytoplasmic fraction. Nuclear extracts were obtained by resuspending the remnants of the pellet in high-salt buffer (the aforementioned hypotonic buffer; $20 \%$ glycerol; $42 \mathrm{mml}^{-1} \mathrm{NaCl}$; and distilled $\mathrm{H}_{2} \mathrm{O}$ ), followed by vigorous tapping for $30 \mathrm{~min}$ and centrifugation at $15000 \times g$ for $5 \mathrm{~min}$ at $4{ }^{\circ} \mathrm{C}$.

After determining the protein concentration of whole cell lysates and nuclear or cytoplasmic extracts by Bradford reagent (Bio-Rad), equal amounts of protein samples were separated by sodium dodecyl sulfate-polyacrylamide gel electrophoresis (SDS-PAGE) and transferred to polyvinylidene difluoride membranes (Millipore, Bedford, MA, USA). The membrane was blocked with either 5\% skimmed milk or bovine serum albumin, then incubated with the aforementioned antibodies overnight at $4{ }^{\circ} \mathrm{C}$. Immunoblots were visualized using the enhanced chemiluminescence detection system (Amersham Pharmacia Biotech, Uppsala, Sweden).

\section{MTT assay}

Cell viability was monitored by the 2-(4,5-dimethylthiazol-2-yl)-2,5diphenyl tetrazolium bromide (MTT) colorimetric assay (Sigma). Briefly, $20 \mu \mathrm{l}$ of MTT $\left(5 \mathrm{mg} \mathrm{ml}^{-1}\right)$ was added to each well. After $4 \mathrm{~h}$ incubation at $37^{\circ} \mathrm{C}$, the cell supernatants were not discarded. MTT crystals were dissolved in dimethyl sulfoxide, and the absorbance was measured at $570 \mathrm{~nm}$. All experiments were performed in 96 well plates and repeated at least three times.

\section{Matrigel invasion assay}

Invasion assays were conducted using modified Boyden chambers with a polycarbonate nucleopore membrane (Corning Costar, Tewksbury, MA, USA). The filter was coated with $10 \mu \mathrm{g}$ Matrigel. The lower surface of the filters was coated with laminin as a chemoattractant. Cells were seeded in duplicate at a density of $2 \times 10^{5}$ cells in RPMI1640 media containing $10 \%$ fetal bovine serum, on the upper compartment of the transwell. The lower compartment was filled with RPMI-1640 media containing 10\% fetal bovine serum, plus $2 \mu \mathrm{g}$ laminin and $0.1 \%$ bovine serum albumin as a chemoattractant. ${ }^{17}$

After incubation for $24 \mathrm{~h}$ at $37^{\circ} \mathrm{C}$, the filters were removed and any cells in the upper surface that did not penetrate the filter were completely wiped out with a cotton swab. Then, the cells that migrated to the lower surface were fixed with methanol, stained with hematoxylin and counted in five randomly selected microscopic fields per filter $(\times 200)$. The average number of counted cells from three independent experiments was represented.

\section{Gelatin zymography}

Conditioned medium and cell lysates were electrophoresed in a polyacrylamide gel containing $1 \mathrm{mg} \mathrm{ml}^{-1}$ of gelatin. Proteolysis was detected as the white zone in a dark blue field, as described previously (J Biol Chem 277, 16396-16402).

\section{Inhibitor study of p38 MAPK, PI3K/AKT and FAK}

For inhibitor studies, we used SB203580 (Calbiochem, La Jolla Diego, CA, USA), LY294002 (Calbiochem), or PF-573228 (Sigma). The cells were pretreated with one of these inhibitors for $1 \mathrm{~h}$ and then transfected with either the syntenin expression vector or empty pCMV-Tag2 vector. Dimethyl sulfoxide was used as a solvent to dissolve SB203580, LY294002 and PF-573228 and as negative control for comparison.

\section{Electrophoretic mobile shift assay}

Nuclear extracts were prepared as described above from cells transfected with either the syntenin vector or empty vector. An electrophoretic mobile shift assay was performed as previously described. ${ }^{18}$ Briefly, $5 \mu \mathrm{g}$ of nuclear extracts were incubated for 30 min with $35 \mathrm{pmol}$ of the ${ }^{32} \mathrm{P}$ end-labeled SP1-specific oligonucleotide $5^{\prime}$-ATTCGATCGGGGCGGGGCGAGC-3' (Santa Cruz Biotechnology) in $10 \mu \mathrm{l}$ binding buffer. The SP1-DNA complex was separated from free oligonucleotides on a $5 \%$ native polyacrylamide gel. The specificity of binding was confirmed by competition with unlabeled oligonucleotides and SP1 antibody (Santa Cruz Biotechnology).

\section{Tumor tissue samples}

The pattern of syntenin expression in neuroendocrine tumors was investigated using a newly manufactured tissue microarray block. We collected a set of neuroendocrine tumors, consisting of 24 small cell carcinomas, 20 carcinoids and 15 neuroblastomas. Small cell carcinoma cases were retrieved from tissue samples from various organs such as lung, stomach, urinary bladder, prostate and liver. The 20 carcinoids were composed of 16 gastrointestinal and 4 pulmonary carcinoids (Table 1). Neuroblastomas were originated from variable sites including the adrenal gland, mediastinum and retroperitoneum. All cases were diagnosed at Seoul National University Hospital from 2004 to 2008. Core tissues ( $2 \mathrm{~mm}$ diameter) were taken from

Table 1 Expression of syntenin in neuroendocrine tumors

\begin{tabular}{|c|c|c|c|}
\hline & $\begin{array}{c}\text { Syntenin } \\
(+)\end{array}$ & $\begin{array}{c}\text { Syntenin } \\
(-)\end{array}$ & \\
\hline Small cell carcinoma $(n=24)$ & $13(54.2 \%)$ & $11(46.8 \%)$ & $\begin{array}{c}P=0.003^{\mathrm{a}}, \\
0.017^{\mathrm{b}}\end{array}$ \\
\hline Lung & 6 & 9 & \\
\hline Stomach & 3 & 1 & \\
\hline Urinary bladder & 0 & 1 & \\
\hline Prostate & 1 & 0 & \\
\hline Liver & 1 & 0 & \\
\hline Tonsil & 1 & 0 & \\
\hline Gall bladder & 1 & 0 & \\
\hline Carcinoid $(n=20)$ & $2(10.0 \%)$ & $18(90.0 \%)$ & \\
\hline Gastrointestinal & 2 & 14 & \\
\hline Pulmonary & 0 & 4 & \\
\hline Neuroblastoma $(n=15)$ & $2(13.3 \%)$ & $13(86.7 \%)$ & \\
\hline
\end{tabular}

aFrequencies of syntenin expression are compared between small cell carcinoma and carcinoid.

b Frequencies of syntenin expression are compared between small cell carcinoma and neuroblastoma. 
formalin-fixed, paraffin-embedded blocks of 59 neuroendocrine tumors (the donor blocks) and arranged in a new recipient paraffin block using the trephine apparatus (Superbiochips Laboratories, Seoul, Korea).

Second, 58 additional SCLC biopsy samples diagnosed at Seoul National University Hospital from 2004 to 2008 were collected and clinically classified into extensive disease and limited disease , according to the criteria from the Veterans' Administration Lung Study Group. ${ }^{19}$ In brief, limited disease was characterized by tumors confined to one hemithorax, whereas all other cases were classified as extensive disease.

\section{Immunohistochemistry}

Immunohistochemical staining for syntenin was conducted using the anti-syntenin murine monoclonal antibody (Abnova). After heatinduced antigen retrieval in citrate buffer $(\mathrm{pH}$ 6.0), sections were incubated with a 1:100 dilution of anti-syntenin antibody for $15 \mathrm{~min}$ at room temperature.

\section{Statistical analysis}

Either Pearson's $X^{2}$ test or Fisher's exact test were used to examine differences between the examined variables. $P$-values of $<0.05$ were considered statistically significant, and all $P$-values were two-sided. All statistical analyses were carried out using SPSS/PC version 12.0 for Windows (SPSS Inc., Chicago, IL, USA).

\section{RESULTS}

Syntenin expression in neuroendocrine tumor tissues, small cell lung cancer tissues and small cell lung cancer cell lines Syntenin was expressed in 13 of 24 small cell carcinomas (54.2\%), a much higher rate than in other neuroendocrine tumors, such as carcinoids $(2 / 20 ; 10.0 \%)$ and neuroblastomas $(2 / 15 ; 13.3 \%)$. These differences were statistically significant (small cell carcinoma vs carcinoid, $P=0.003$; small cell carcinoma vs neuroblastoma, $P=0.017$ ) (Table 1). In the positive cases, syntenin was strongly expressed in the cell membrane and cytoplasm (Figure 1). There was no significant difference in syntenin expression among small cell carcinomas from variable organs. Cases with syntenin expression showed poor clinical outcomes, as multiple metastases at diagnosis or expiration occurred more frequently than in cases without syntenin expression (7/13 (53.8\%) in syntenin-positive vs $3 / 11$ $(27.3 \%)$ in syntenin-negative cases). This difference, however, was not statistically significant.

In 58 SCLCs evaluated in biopsy tissues, syntenin was expressed in 9 of 58 cases $(15.5 \%)$. Cases with syntenin expression had more extensive and advanced disease at diagnosis as compared with syntenin-negative cases $(P=0.029)$ (Table 2).

Among the six SCLC cell lines, syntenin expression was low in NCI-H187 and NCI-H417 (the syntenin-low cell lines) and high in NCI-H69, NCI-H146, NCI-H209 and NCI-H889 (the syntenin-high cell lines) at both the protein and mRNA levels (Figures 1e and f). NCI-H187 and H69 cells were subjected to further functional studies.

\section{Overexpression of syntenin increases cell viability, proliferative activities and invasiveness in SCLC cells} We transfected the FLAG-tagged syntenin expression vector or empty vector into the syntenin-low NCI-H187 cells. Western blotting showed a lower band $(33 \mathrm{kDa})$ corresponding to endogenous syntenin and an upper band $(34 \mathrm{kDa})$ corresponding to exogenously transfected FLAG-tagged

\section{Table 2 Expression of syntenin in small cell lung cancer}

\begin{tabular}{lccc}
\hline & Syntenin $(+)$ & Syntenin $(-)$ & \\
\hline Limited disease & 1 & 26 & $P=0.029$ \\
Extensive disease & 8 & 23 & \\
\hline
\end{tabular}
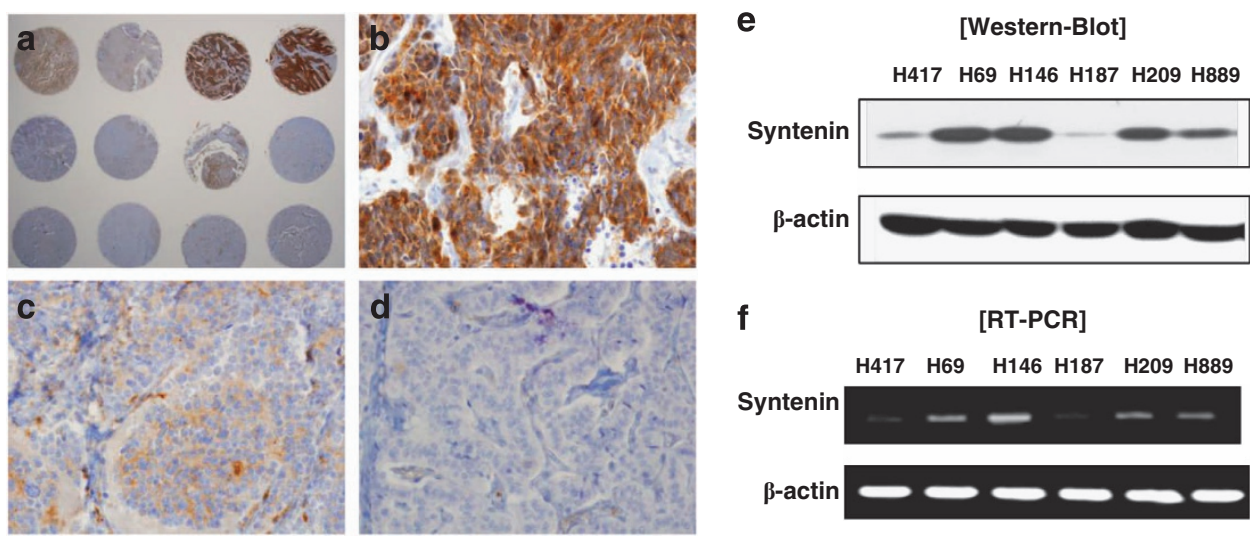

Figure 1 Expression of syntenin in neuroendocrine tumors and basal expression levels of syntenin in small cell lung cancer cell lines. (a) Immunohistochemical staining of syntenin in an array block of neuroendocrine tumors shows strong expression in small cell carcinomas. Representative examples of small cell carcinoma, neuroblastoma and carcinoid are shown in $\mathbf{b}$, c and $\mathbf{d}$, respectively. (e) Western blot analysis showed that syntenin is expressed at relatively low levels in $\mathrm{NCl}-\mathrm{H} 187$ and $\mathrm{NCl}-\mathrm{H} 417$ cells. In contrast, other cell lines, such as $\mathrm{NCl}-\mathrm{H} 69, \mathrm{NCl}-\mathrm{H} 146, \mathrm{NCl}-\mathrm{H} 209$ and $\mathrm{NCl}-\mathrm{H} 889$, showed relatively high syntenin expression. (f) RT-PCR analysis for comparison of syntenin at the mRNA level showed relatively high mRNA levels in NCl-H69, NCl-H146, NCl-H209 and NCl-H889 cells, but low levels in $\mathrm{NCl}-\mathrm{H} 187$ and $\mathrm{NCl}-\mathrm{H} 417$ cells. 
syntenin (Figure 2a). RT-PCR also showed marked upregulation of syntenin mRNA in NCI-187 cells transfected with the syntenin expression vector (Figure 2c). However, synteninhigh NCI-H69 cells were transfected with either syntenin siRNA or scrambled siRNA, and syntenin siRNA effectively inhibited syntenin expression on both the protein and mRNA levels (Figures $2 \mathrm{~b}$ and $\mathrm{c}$ ).

Using these cell lines, we first analyzed the effect of syntenin on cell viability and proliferation by MTT assay. Cell viability and proliferation were enhanced by forced expression of syntenin in NCI-H187 cells and inhibited when syntenin was downregulated by siRNA in NCI-H69 cells (Figure 2d).

Next, we used a Matrigel invasion assay to determine the effect of syntenin on the invasiveness of SCLC cells. As shown in Figure 2e, the number of cells that invaded into the lower surface of the filter was about five to six times higher in syntenin-transfected NCI-H187 cells as compared with empty vector-transfected cells. When syntenin expression was downregulated by siRNA in NCI-H69 cells, the number of invaded cells decreased (Figure 2e). These results suggest that syntenin expression might have a role in the invasiveness of SCLC.

\section{Syntenin induces MMP2 and MT1-MMP, but not MMP9, expression in SCLC cells}

To determine if syntenin increases the invasiveness of SCLC cells by inducing MMPs and what type of MMPs are involved, cell lysates or supernatants of syntenin-transfected NCI-H187 cells or syntenin siRNA-transfected NCI-H69 cells were collected and used for western blot analysis. In the supernatant of syntenin-transfected NCI-H187 cells, active MMP2 protein levels $(63 \mathrm{kDa}$ in size) were increased, compared with empty vector-transfected cells, proportionate to the degree of the serial concentration of supernatant (Figure 3a). In syntenin-transfected cells, a marked increase of the a

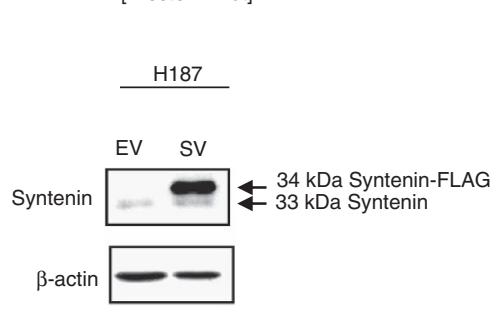

d

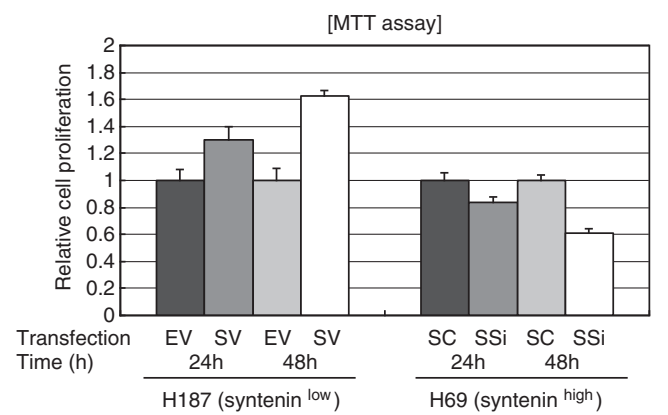

EV : empty vector SV : syntenin vector b

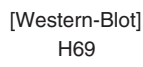

H69

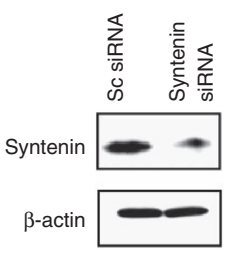

C

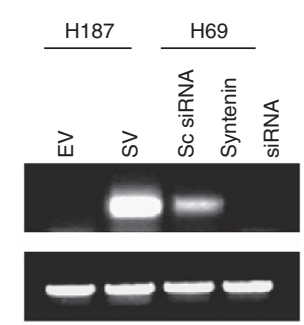

e

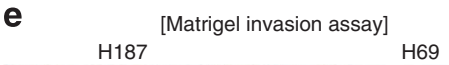

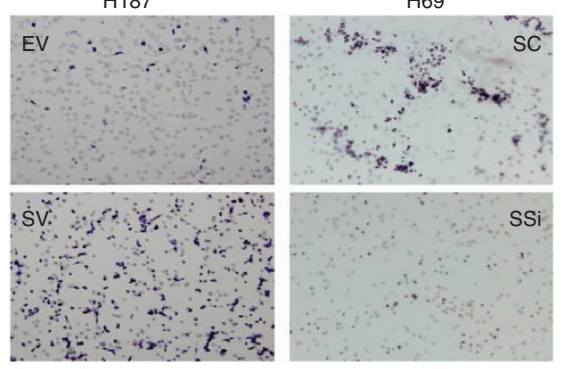
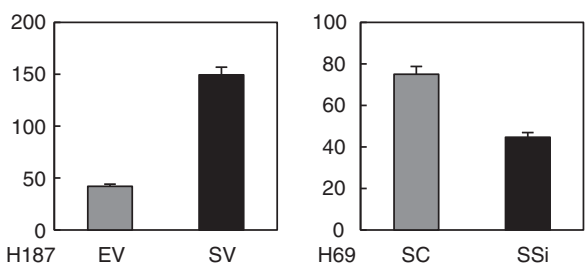

Figure 2 Effect of either forced expression or inhibition of syntenin in small cell lung cancer cells on expression levels, proliferation activities and invasiveness. (a) By transfecting a FLAG-tagged syntenin expression vector in $\mathrm{NCl}-\mathrm{H} 187$ cells, two bands, $33 \mathrm{kDa}$ and $34 \mathrm{kDa}$ in size, were observed via western blotting. This difference of band size was derived from increased protein size, due to the FLAG tag. (b) Transfection of syntenin siRNA in NCl-H69 cells revealed a significant reduction of syntenin expression. (c) RT-PCR showed a corresponding change in syntenin mRNA levels after syntenin overexpression or inhibition. (d) In the MTT assay, cell viability and proliferation activity were enhanced by forced expression of syntenin vector, but inhibited by transfection of syntenin siRNA, compared with negative controls, respectively. (e) In the hematoxylin-stained lower surface of filters harvested after $24 \mathrm{~h}$, $\mathrm{NCl}-\mathrm{H} 187 \mathrm{cells}$ transfected with the syntenin vector were observed five to six times more than empty vector-transfected cells (top left). In contrast, syntenin inhibition by siRNA decreased migration or invasion in $\mathrm{NCl}-\mathrm{H} 69$ cells (top right). The average number of invaded cells from five randomly selected fields per filter was plotted in the error bars (bottom). MTT, 2-(4,5-dimethylthiazol-2-yl)-2,5-diphenyl tetrazolium bromide. 
a

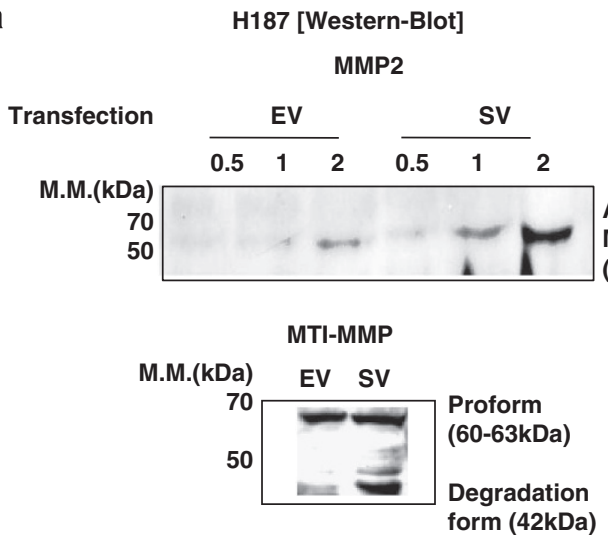

C

H187 [Gelatin Zymography]

MMP2

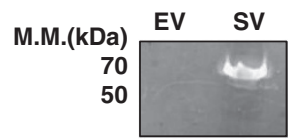

[RT-PCR]

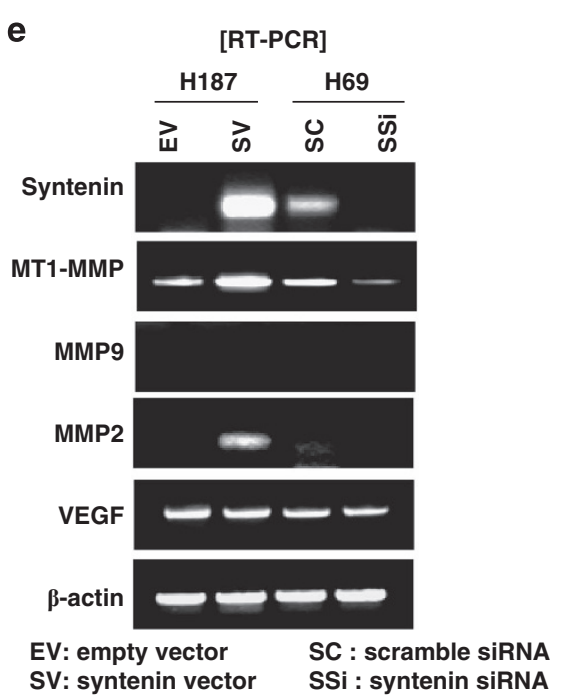

b

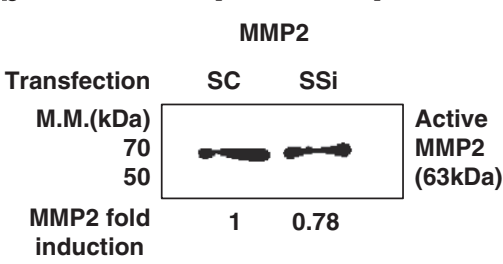

MTI-MMP

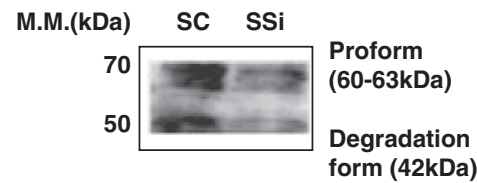

d H69 [Gelatin Zymography]

MMP2

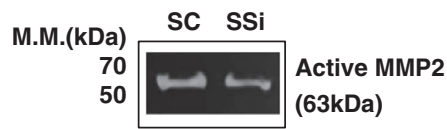

(63kDa)
Pro-MMP2 (72kDa)

Active MMP2 (63kDa)
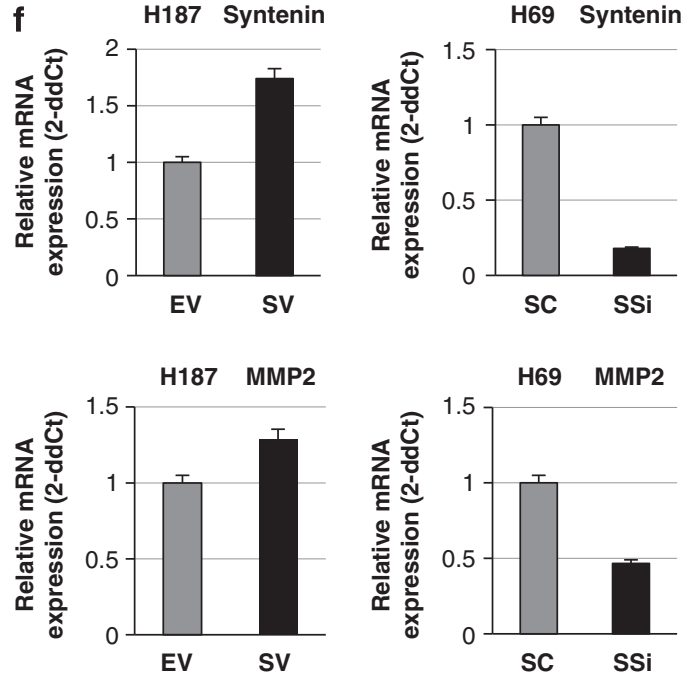

Figure 3 Effects on invasiveness-associated molecules elicited by syntenin overexpression in syntenin-low NCl-H187 cells and syntenin knockdown in syntenin-high $\mathrm{NCl}-\mathrm{H} 69$ cells. (a, top) In the concentrated supernatant of syntenin-transfected $\mathrm{NCl}-\mathrm{H} 187$ cells, the active form of MMP2 was identified via western blot and reconfirmed by serial doubling of the loading dose. (a, bottom) In a western blot of whole cell lysates, a slight augmentation of the pro-form band of $60-63 \mathrm{kDa}$ and a marked increase of the degradation form of $42 \mathrm{kDa}$ of MT1-MMP were observed. (b, top) The active form of MMP2 was decreased in the supernatant of syntenin-inhibited NCl-H69 cells. (b, bottom) In a western blot of whole cell lysates, the pro-form and the degradation form of MT1-MMP were reduced following syntenin inhibition. (c) Gelatin zymography analysis showed MMP2 activity in the conditioned medium harvested from empty vector or syntenin vector-transfected $\mathrm{NCl}-\mathrm{H} 187$ cells. The $72-\mathrm{kDa}$ band was identified in syntenin-overexpressed $\mathrm{NCl}-\mathrm{H} 187$ cells. This band corresponds to the previously known pro-form size of MMP2. The active form band $(63 \mathrm{kDa})$ was also observed below the pro-form band. (d) In gelatin zymography analysis, syntenin siRNA-transfected $\mathrm{NCl}-\mathrm{H} 69$ cells displayed decreased expression of active MMP2 in the conditioned medium. (e) RT-PCR analysis showed that both MTI-MMP and MMP2 mRNA were induced by syntenin overexpression in NCl-H187 cells. In contrast, significant inhibition of MT1-MMP mRNA was found by transfection of syntenin siRNA in NCl-H69 cells. (f) The expression of syntenin and MMP2 in $\mathrm{NCl}-\mathrm{H} 187$ and $\mathrm{H} 69$ cells under circumstances of syntenin overexpression or inhibition were verified by quantitative real-time RT-PCR, the results of which were consistent with the results of RT-PCR analysis. MMP, matrix metalloproteinase; MT1, membrane type 1; VEGF, vascular endothelial growth factor.

degraded form of membrane type 1-matrix metalloproteinase (MT1-MMP) was identified by western blot using whole cell lysates (Figure 3a), with a slight increase in the pro-form $(60-63 \mathrm{kDa})$. This suggests that MT1-MMP enzyme activity was increased by the overexpression of syntenin. In contrast, syntenin siRNA-transfected NCI-H69 cells showed decreased active MMP2 in the supernatant, compared with scrambled siRNA-transfected cells (Figure $3 \mathrm{~b}$ ). The decrease in the 
degraded form of MT1-MMP was also noted in a western blot using whole cell lysates (Figure 3b), with a slight decrease in the pro-form.

To further ascertain the induction of functional MMP2 by syntenin overexpression, we performed gelatin zymography. As shown in Figure $3 \mathrm{c}$, the augmented bands of $72 \mathrm{kDa}$ and $63 \mathrm{kDa}$ in size were observed in syntenin-overexpressed NCIH187 cells. These bands correspond to the pro-form and active form of MMP2, respectively. In contrast, syntenin siRNAtransfected NCI-H69 cells displayed decreased expression of active MMP2 in conditioned medium (Figure 3d). These data indicate that MMP2 is induced and also activated by syntenin, which might underlie the role of syntenin in promoting the invasiveness of SCLC cells.

Syntenin-mediated induction of MT1-MMP and MMP2 could occur at the transcriptional level and/or post-transcriptional level. To address this issue, total RNA was extracted and RT-PCR was conducted for invasiveness-associated molecules including MTI-MMP, MMP2, MMP9 and vascular endothelial growth factor (VEGF) (Figure 3e). RT-PCR analysis showed that both MTI-MMP and MMP2 mRNAs were induced by syntenin overexpression in NCI-H187 cells. In contrast, in endogenously syntenin-high NCI-H69 cells, significant inhibition of MT1-MMP mRNA was found after transfection with syntenin siRNA. The expression of syntenin and MMP2 in NCI-H187 and H69 cells under circumstances of either syntenin overexpression or inhibition were double-checked by quantitative real-time RT-PCR. These data were consistent with the results of RT-PCR analysis (Figure 3f). These results suggest that syntenin-mediated signaling may be transduced to the nucleus, leading to upregulation of MTI-MMP and MMP2 gene expression. The levels of VEGF and MMP9 mRNAs were not changed by syntenin in both cell lines.

\section{Overexpression of syntenin activates the p38 mitogen-} activated protein kinase and AKT pathways in SCLC, along with focal adhesion kinase activation, without interaction with the extracellular matrix

To reveal what signaling pathways are involved in the synteninmediated induction of MT1-MMP and MMP2 in SCLC, we investigated the alteration of p38 mitogen-activated protein kinase (MAPK), AKT and c-jun activity after syntenin overexpression and knockdown. These pathways were selected on the basis of previous studies on syntenin., ${ }^{9,11,20}$ In NCI-H187 cells, western blot analysis showed that phosphorylation of p38 MAPK and AKT increased after syntenin overexpression (Figure 4a). Moreover, AKT phosphorylation was decreased after transfection of syntenin siRNA in NCI-H69 cells. A slight reduction in p38 phosphorylation was also observed in syntenin siRNA-transfected NCI-H69 cells. However, c-jun phosphorylation was not affected by syntenin expression in both NCI-H187 and H69 cells.

In previous studies, ${ }^{9,13}$ syntenin overexpression also induced activation of FAK, which has a role in initiating the activation of integrin-mediated signaling pathways via interaction with ECM proteins such as fibronectin and collagen. In our study,
SCLC cells were grown in suspension without ECM interaction. Therefore, we determined whether syntenin overexpression could induce FAK activation in the absence of ECM interaction. Increased FAK phosphorylation was observed in syntenin-overexpressed NCI-H187 cells, whereas FAK phosphorylation was decreased in syntenin-inhibited NCI-H69 cells. These results indicate that in SCLC, syntenin alone can activate FAK and likely subsequent signaling pathways, even in conditions lacking ECM stimulation (Figure 4a).

\section{Syntenin induces MT1-MMP and MMP2 through activation} of focal adhesion kinase, p38 MAPK and PI3K/AKT pathways

To confirm whether the signaling pathways activated by syntenin are associated with increased invasiveness, we evaluated the influence of a p38 inhibitor (SB203580), a PI3K/ AKT inhibitor (LY294002) and a FAK inhibitor (PF-573228) on MT1-MMP and MMP2 expression in syntenin-overexpressed cells. The optimal concentration $(10 \mu \mathrm{M})$ of SB203580 was determined by relative comparison of phosphorylated-p38 (p-p38) levels between empty vector-transfected and syntenin vector-transfected condition. At this concentration, p-p38 levels were slightly decreased, in spite of syntenin overexpression in syntenin-transfected cells, although the basal level of p-p38 was upregulated in empty vector-transfected cells, due to the role of SB203580 as not only inhibitor but also as a partial agonist. In contrast, p-p38 was increased in synteninoverexpressed cells treated with dimethyl sulfoxide as a control. Pharmacological inhibition of the p38 MAPK pathway led to a marked reduction of MMP2 and MT1-MMP mRNA levels, despite syntenin overexpression (Figure $4 \mathrm{~b}$ ).

Similarly, inhibition of the PI3K/AKT pathway by LY294002 $(1 \mu \mathrm{M})$ or FAK by PF-573228 $(1 \mu \mathrm{M})$ antagonized the synteninmediated upregulation of MMP2 and MT1-MMP, and rather led to downregulation of MMP2 and MT1-MMP, despite syntenin overexpression (Figures $4 \mathrm{c}$ and $\mathrm{d}$ ).

When FAK, p38 and PI3K/AKT were pharmacologically inhibited in NCI-H187 cells, the number of invaded cells in the Matrigel invasion assay did not increase in syntenin-transfected cells, compared with empty vector-transfected cells (Figure 4e). In NCI-H69 cells, the number of invaded cells decreased after inhibition of $\mathrm{p} 38$ and PI3K/AKT (Figure 4f). In addition, the invasiveness of NCI-H69 cells was more suppressed by syntenin inhibition.

Together, these results suggest that activation of FAK and the p38 MAPK and PI3K/AKT pathways are associated with invasiveness-related syntenin signaling transduction, leading to upregulation of MMP2 and MT1-MMP.

\section{Syntenin functions by activating the SP1 transcription} factor in SCLC, but has little effect on NF- $\kappa B$ activity Previous reports using malignant melanoma cells showed that NF- $\kappa B$ activity was upregulated by forced expression of

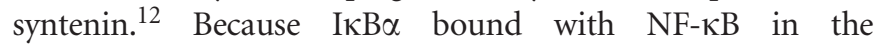
cytoplasm is degraded as cytosolic NF- $\kappa B$ moves to 


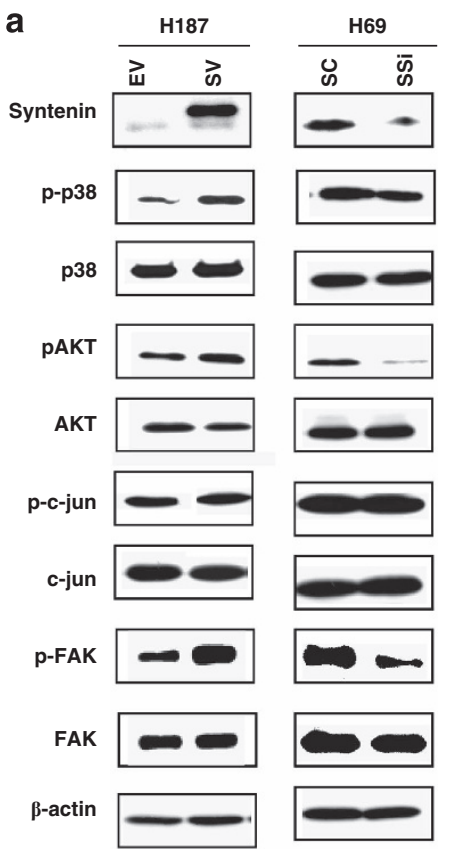

b
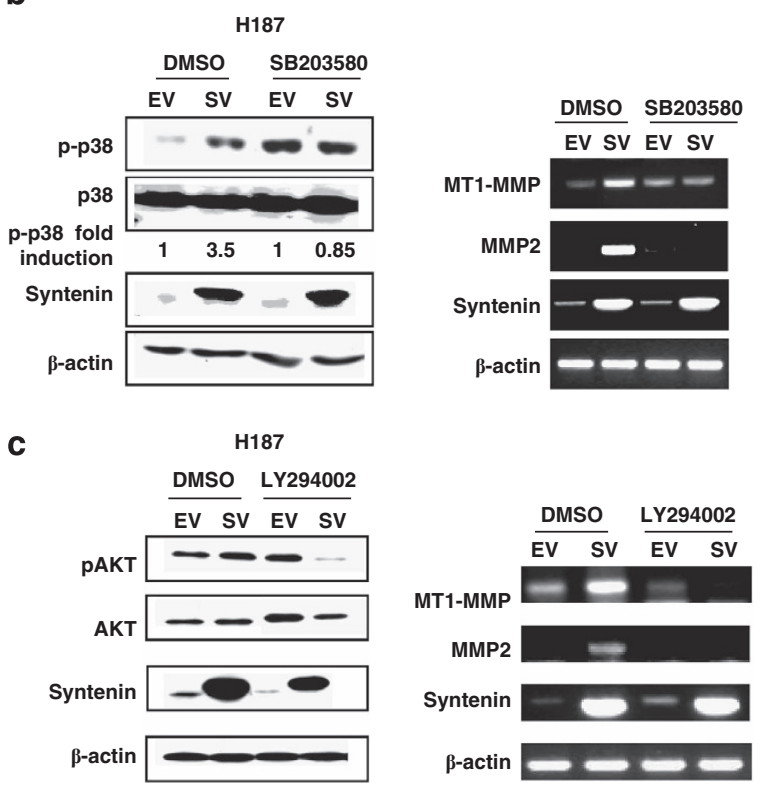

d

H187
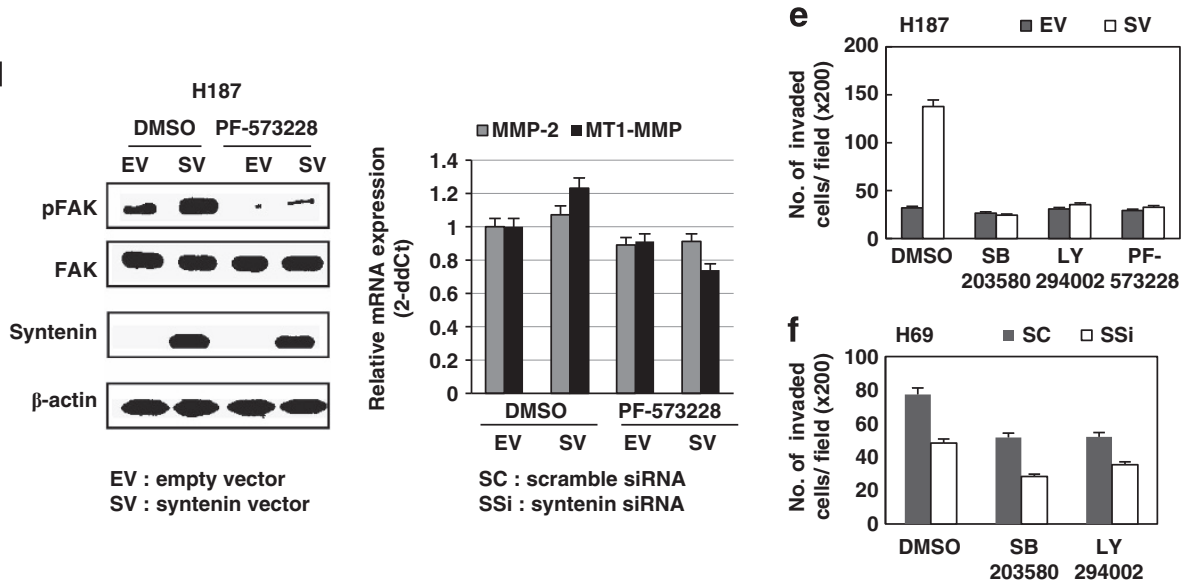

Figure 4 Activation of p38, AKT and focal adhesion kinase by forced expression of syntenin in small cell lung cancer cells. (a) Western blot analysis revealed enhanced phosphorylation of p38 MAPK and AKT after syntenin overexpression in $\mathrm{NCl}-\mathrm{H} 187$ cells, compared with empty-vector transfection. In contrast, phosphorylated AKT and p38 MAPK were shown to be decreased by syntenin inhibition in NCI$\mathrm{H} 69$ cells. Activation of focal adhesion kinase (FAK) by syntenin overexpression and suppression of FAK by syntenin inhibition was also observed without stimulation of any extracellular matrix proteins. (b) Western blot analysis after administration of SB203580 (10 $\mu \mathrm{m}$ ) revealed the inhibition of p38 activation in syntenin-transfected cells compared with the p38 level in empty vector-transfected cells, although the basal level itself was upregulated, due to the role of SB203580 as not only an inhibitor but also as a partial agonist (left). In RT-PCR analysis, the inhibition of p38 MAPK induced the inhibition of both MMP2 and MT1-MMP mRNA (right). (c) The administration of LY294002 (1 $\mu \mathrm{M})$ suppressed AKT activation in syntenin-transfected cells (left), which led to the downregulation of MMP2 and MT1-MMP, despite syntenin overexpression (right). (d) The activation of FAK was inhibited by administration of PF-573228 (1 $\mu \mathrm{M})$ (left), which antagonized the syntenin-mediated upregulation of MMP2 and MT1-MMP (right). (e) When FAK, P38 and PI3K/AKT were inhibited by pharmacological inhibitors in $\mathrm{NCl}-\mathrm{H} 187$ cells, the number of invaded cells in the Matrigel invasion assay did not increase in syntenin-transfected cells. (f) In NCl-H69 cells, the number of invaded cells decreased after inhibition of p38 and PI3K/AKT. The invasiveness of $\mathrm{NCl}-\mathrm{H} 69$ cells was suppressed by syntenin inhibition. MAPK, mitogen-activated protein kinase; MMP, matrix metalloproteinase; MT1, membrane type 1.

the nucleus to be activated, we assessed the degradation of IкB $\alpha$ in syntenin-overexpressed NCI-H187 cells to determine whether NF- $\kappa B$ activity is regulated by syntenin in SCLC. The levels of IKB $\alpha$ analyzed at $12 \mathrm{~h}, 18 \mathrm{~h}$ and $24 \mathrm{~h}$ after syntenin transfection were not changed (Figure 5a). Moreover, we measured and compared the levels of the p65 subunit of $\mathrm{NF}-\kappa \mathrm{B}$ between the cytosolic and nuclear extracts in synteninor empty vector-transfected NCI-H187 cells. As shown in Figure 5b, the cytoplasmic levels of p65 were not altered by syntenin overexpression, and nuclear translocation of p65 was 
a

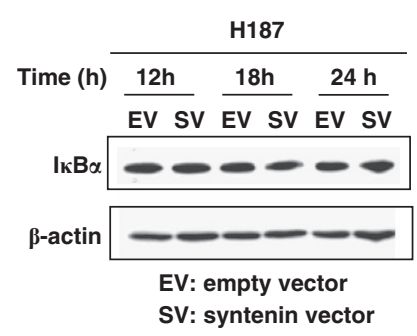

b

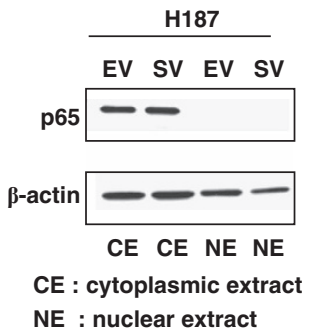

C
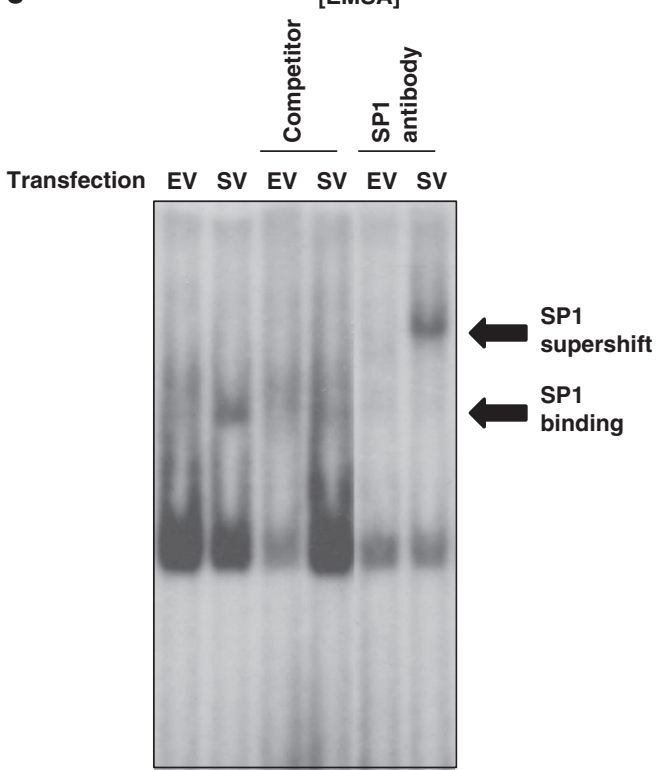

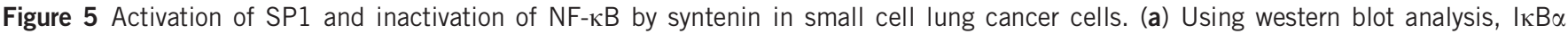
levels in whole cell lysates were analyzed three times, at $12 \mathrm{~h}, 18 \mathrm{~h}$ and $24 \mathrm{~h}$ after transfection. Time-dependent degradation was not observed. (b) The cytoplasmic levels of p65 were not altered by syntenin overexpression, compared with empty vector transfection, and nuclear translocation of p65 was not identified. (c) In EMSA assay using an SP1 consensus oligomer labeled with ${ }^{32}$, the SP1 binding activity was upregulated in syntenin-transfected cells. Adding an unlabeled SP1-specific oligonucleotide (cold competitor) caused decreased binding. Incubation with an SP1 antibody revealed a super-shifted band, due to the increased molecular weight of the DNA-protein complex produced by antibody binding. This revealed that SP1 intervened in syntenin-induced signaling. EMSA, electrophoretic mobile shift assay; FAK, focal adhesion kinase; MMP, matrix metalloproteinase; MT1, membrane type 1.

not observed. These results suggest that NF- $\mathrm{B}$ might not be involved in the syntenin signaling pathway in SCLC cells.

Because NF- $\kappa \mathrm{B}$ activity was not affected by syntenin activation, we searched for other molecules that transduce signals to the nucleus. SP1 was chosen because this transcription factor binds both MMP2 and MT1-MMP gene promoter regions. $^{21}$ Using electrophoretic mobile shift assay, we observed a marked increase in SP1 binding activity in the nuclear extracts from syntenin-overexpressed NCI-H187 cells, compared with empty vector-transfected cells (Figure 5c). The binding specificity of the DNA-protein complex was shown by the decrease in SP1 binding, through interaction with an unlabeled cold competitor. Incubation with an SP1 antibody showed a super-shifted band, again reconfirming the specific binding between SP1 and the consensus oligomer (Figure $5 \mathrm{c}$ ). These results suggest that SP1 is activated by syntenin and might function as an important transcription factor in syntenin-related signaling to promote invasiveness in SCLC cells.

\section{DISCUSSION}

This study was designed to clarify the invasiveness-associated role of syntenin in SCLC. For this purpose, we performed immunohistochemical staining for syntenin on human SCLC tissues and carried out functional studies using SCLC cell lines.

In immunohistochemical analyses of 58 SCLC tissues, syntenin was expressed in nine cases (15.5\%). Cases with syntenin expression had more extensive and advanced disease at the time of diagnosis. These results suggest that there may be a subset of SCLCs characterized by syntenin expression and its association with more advanced tumor invasion. Furthermore, the rate of syntenin expression in small cell carcinomas of various sites including lung was much higher than in other neuroendocrine tumors, such as carcinoids and neuroblastomas, suggesting that syntenin expression may relate to more aggressive forms of neuroendocrine tumors. When SCLC cell lines were classified into two categories (syntenin-high and syntenin-low cells) according to syntenin expression levels, syntenin overexpression in syntenin-low cells increased the viability, proliferation and invasiveness of SCLC cells and induced expression of both MT1-MMP and MMP2, important ECM-degrading proteins. In syntenin-high cells, syntenin inhibition resulted in decreased cellular invasion along with significant reductions of MT1-MMP and MMP2. The present results suggest that syntenin may regulate the expression of ECM-degrading MMPs and act as an important molecule in tumor invasiveness of SCLC.

This study showed MT1-MMP and MMP2 induction as a final product of syntenin overexpression in SCLC cells. The literature review showed that MMP2 was not highly expressed in several SCLC cell lines and SCLC tissues. ${ }^{22-25}$ According to previous reports, MMP1, 9, 11, 13 and 14 (that is, MT1MMP) are the frequently expressed MMPs in SCLC. Therefore, the induction of MT1-MMP, another product of syntenin activation, could have more biological and clinical relevance in SCLC. In a previous report, MT1-MMP overexpression, 
among several MMPs, was shown to have the strongest prognostic implications in SCLC. ${ }^{22}$

The syntenin-mediated signaling pathway is interesting in that it is differentially activated by different types of ECM stimulation among variable cancer cell lines. In malignant melanoma cell lines stimulated with fibronectin, syntenin activated the p38 MAPK pathway as well as NF- $\kappa B^{2,9,12}$ In breast cancer cell lines activated with type 1 collagen, syntenin activated the PI3K/AKT and ERK pathways, along with activation of RhoA, cdc42, Racl and H-ras. ${ }^{11}$ In our study, SCLC cells showed activation of p38 MAPK, PI3K/AKT and SP1 after syntenin overexpression. The dual activation of $\mathrm{p} 38$ MAPK and PI3K/AKT pathway is unique, compared with previous studies. ${ }^{9,11}$ The role of SP1 as a transcription factor in syntenin-mediated signaling was first demonstrated in our study. In contrast, we did not observe any effect of syntenin on NF- $\kappa \mathrm{B}$ activity in SCLC cells, although previous studies emphasized the role of $\mathrm{NF}-\kappa \mathrm{B}$ in syntenin-related signaling pathways. ${ }^{12,26}$ These findings suggest that syntenin, an adaptor protein with various binding motifs, could utilize variable signaling molecules to transduce signals, depending on the cell type.

Another unique finding in our study is that downstream signal transduction of syntenin in SCLC cells was initiated in the absence of the ECM interaction via integrin molecules that is observed in other tumors. ${ }^{27,28}$ In previous studies on syntenin, variable ECM stimulation was used. After transfection of syntenin-expressing virus, melanoma cells were harvested and transferred into fibronectin-coated dishes, followed by recognition of various changes in the signaling molecules and induction of MMPs., ${ }^{9,12}$ Type 1 collagen was also used as an ECM stimulant in either gastric or breast cancer cell lines. ${ }^{10,11}$ Our study, for the first time, showed that induction of MMPs and signal transduction by syntenin overexpression could occur without ECM stimulation and the ECM-integrin initiating interaction. The precise explanation for the mechanism underlying these differences between previously studied adherent cell lines and SCLC cells with floating growth remains to be clarified. This phenomenon may reflect some biological differences between adherent cells and suspension cells. One speculative explanation could be the ECM-unmediated activation of integrins via auto-activation of FAK, which colocalizes with syntenin at the cell membrane and transfers the signal from integrin to syntenin.

In this study, we used suspension SCLC cells, showing an anchorage-independent growth pattern without ECM stimulation. The influence of ECM interaction has been analyzed in several studies in various aspects, including functional and morphological changes. Adhesion of SCLC cells with ECM proteins enhances the tumorigenicity and confers resistance to chemotherapeutic agents, as a result of $\beta 1$-integrin-stimulated signaling pathway suppressing chemotherapy-induced apoptosis. ${ }^{16,17,29}$ In addition to functional augmentation of ECM interactions, morphologic alterations of SCLC cells after adhesion of ECM matrix proteins were also shown in several previous reports. ${ }^{16,17}$ These results suggest that ECM stimulation might be associated with the activation of various signaling pathways through ECM-cell interactions including integrin-linked signaling and this activation might enhance the adaptive response of SCLC cells under certain circumstances, including the administration of chemotherapeutic agents, thereby promoting the malignant phenotype and cell survival. Because SCLC cells are surrounded by an extensive stroma of ECM in vivo, ${ }^{16}$ these ECM-cell interactions could have more significant meaning in SCLC cells. In these respects, the results of syntenin expression without ECM stimulation in our in vitro study suggest that syntenin might have a more significant role in terms of invasiveness in vivo, where more complicated interactions with the ECM also occur. Furthermore, it is noteworthy that in the Matrigel invasion assay in our study, the basic conditions were associated with a laminin-associated chemoattractant effect and that cell invasion was not initiated without laminin stimulation.

Taking the results about syntenin-mediated signaling in this study together, we present a hypothetical model in Figure 6. Without extracellular matrix interaction, FAK is able to be phosphorylated by syntenin overexpression in SCLC, subsequently activating signaling pathways including p38 MAPK and AKT. Through the SP1 transcription factor, the

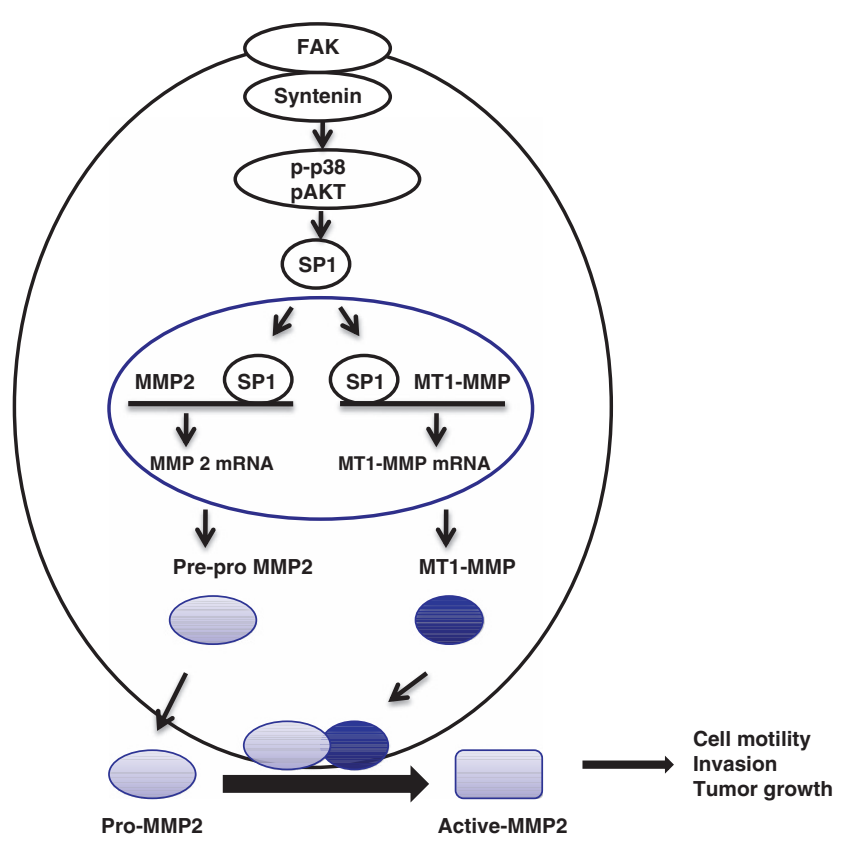

Figure 6 Schematic model of signaling pathways activated by syntenin activation in small cell lung cancer cells. Without extracellular matrix interactions, focal adhesion kinase (FAK) is phosphorylated by syntenin activation. The activated signal is then transduced via p38 MAPK and AKT phosphorylation. Through action of the gene-regulating element SP1, signal transduction progressed from the cytoplasm to nucleus. Finally, expression of MT1-MMP and MMP2 was induced, and these products then enhanced cell motility, invasion and tumor growth. MAPK, mitogen-activated protein kinase; MMP, matrix metalloproteinase; MT1, membrane type 1 . 
signal is transduced from the cytoplasm to the nucleus, leading finally to the induction and upregulation of MT1-MMP and MMP2. These products then contribute to the enhancement of cell motility, invasion and tumor growth.

These results suggest that syntenin might contribute to the invasiveness of SCLC via unique pathways, different from other cancer cells, and could be utilized as a new therapeutic target to control tumor invasion and metastasis in SCLC.

\section{CONFLICT OF INTEREST}

The authors declare no conflict of interest.

\section{ACKNOWLEDGEMENTS}

This work was supported by the Global Core Research Center (GCRC) grant (No. 2013-003531) from the National Research Foundation (NRF), Ministry of Education, Science and Technology (MEST), Republic of Korea. The study was conducted at the Tumor Immunity Medical Research Center, Cancer Research Center, Seoul National University College of Medicine, 28 Yeongeon-dong, Jongro-gu, Seoul 110-799, Korea.

1 Grootjans JJ, Reekmans G, Ceulemans H, David G. Syntenin-syndecan binding requires syndecan-synteny and the co-operation of both PDZ domains of syntenin. J Biol Chem 2000; 275: 19933-19941.

2 Sarkar D, Boukerche H, Su ZZ, Fisher PB. mda-9/Syntenin: more than just a simple adaptor protein when it comes to cancer metastasis. Cancer Res 2008; 68: 3087-3093.

3 Zimmermann P, Tomatis D, Rosas M, Grootjans J, Leenaerts I, Degeest G et al. Characterization of syntenin, a syndecan-binding PDZ protein, as a component of cell adhesion sites and microfilaments. Mol Biol Cell 2001; 12: 339-350.

4 Grootjans JJ, Zimmermann P, Reekmans G, Smets A, Degeest G, Durr J et al. Syntenin, a PDZ protein that binds syndecan cytoplasmic domains. Proc Natl Acad Sci USA 1997; 94: 13683-13688.

5 Helmke BM, Polychronidis M, Benner A, Thome M, Arribas J, Deichmann M. Melanoma metastasis is associated with enhanced expression of the syntenin gene. Oncol Rep 2004; 12: 221-228.

6 Sarkar D, Boukerche H, Su ZZ, Fisher PB. mda-9/syntenin: recent insights into a novel cell signaling and metastasis-associated gene. Pharmacol Ther 2004; 104: 101-115.

7 Boukerche H, Baril P, Tabone E, Berard F, Sanhadji K, Balme B et al. A new Mr 55,000 surface protein implicated in melanoma progression: association with a metastatic phenotype. Cancer Res 2000; 60: 5848-5856.

8 Baril P, Nejjari M, Scoazek JY, Boukerche H. Blocking a novel 55 kDa melanoma-associated cell surface antigen inhibits the development of spontaneous metastases and interactions with frozen lung section. Int $J$ Cancer 2002; 99: 315-322.

9 Boukerche H, Su ZZ, Emdad L, Baril P, Balme B, Thomas L et al. mda-9/ Syntenin: a positive regulator of melanoma metastasis. Cancer Res 2005; 65: 10901-10911.

10 Koo TH, Lee JJ, Kim EM, Kim KW, Kim HD, Lee JH. Syntenin is overexpressed and promotes cell migration in metastatic human breast and gastric cancer cell lines. Oncogene 2002; 21: 4080-4088.

11 Meerschaert K, Bruyneel E, De Wever O, Vanloo B, Boucherie C, Bracke M et al. The tandem PDZ domains of syntenin promote cell invasion. Exp Cell Res 2007; 313: 1790-1804.

12 Boukerche H, Su ZZ, Emdad L, Sarkar D, Fisher PB. mda-9/Syntenin regulates the metastatic phenotype in human melanoma cells by activating nuclear factor-kappaB. Cancer Res 2007; 67: 1812-1822.

13 Boukerche H, Su ZZ, Prevot C, Sarkar D, Fisher PB. mda-9/Syntenin promotes metastasis in human melanoma cells by activating c-Src. Proc Natl Acad Sci USA 2008; 105: 15914-15919.

14 Buttery RC, Rintoul RC, Sethi T. Small cell lung cancer: the importance of the extracellular matrix. Int J Biochem Cell Biol 2004; 36: 1154-1160.

15 Nicholson SA, Beasley MB, Brambilla E, Hasleton PS, Colby TV, Sheppard MN et al. Small cell lung carcinoma (SCLC): a clinicopathologic study of 100 cases with surgical specimens. Am J Surg Pathol 2002; 26: 1184-1197

16 Sethi T, Rintoul RC, Moore SM, MacKinnon AC, Salter D, Choo C et al. Extracellular matrix proteins protect small cell lung cancer cells against apoptosis: a mechanism for small cell lung cancer growth and drug resistance in vivo. Nat Med 1999; 5: 662-668.

17 Fridman R, Giaccone G, Kanemoto T, Martin GR, Gazdar AF, Mulshine JL. Reconstituted basement membrane (matrigel) and laminin can enhance the tumorigenicity and the drug resistance of small cell lung cancer cell lines. Proc Natl Acad Sci USA 1990; 87: 6698-6702.

18 Bae IH, Park MJ, Yoon SH, Kang SW, Lee SS, Choi KM et al. Bcl-w promotes gastric cancer cell invasion by inducing matrix metalloproteinase-2 expression via phosphoinositide 3-kinase, Akt, and Sp1. Cancer Res 2006; 66: 4991-4995.

19 Zelen M. Keynote address on biostatistics and data retrieval. Cancer Chemother Rep 3 1973; 4: 31-42.

20 Luyten A, Mortier E, Van Campenhout C, Taelman V, Degeest G, Wuytens G et al. The postsynaptic density 95/disc-large/zona occludens protein syntenin directly interacts with frizzled 7 and supports noncanonical Wnt signaling. Mol Biol Cell 2008; 19: 1594-1604.

21 Overall CM, Lopez-Otin C. Strategies for MMP inhibition in cancer: innovations for the post-trial era. Nat Rev Cancer 2002; 2: 657-672.

22 Michael M, Babic B, Khokha R, Tsao M, Ho J, Pintilie M et al. Expression and prognostic significance of metalloproteinases and their tissue inhibitors in patients with small-cell lung cancer. J Clin Oncol 1999; 17: 1802-1808.

23 Damstrup L, Rude Voldborg B, Spang-Thomsen M, Brunner N, Skovgaard Poulsen $\mathrm{H}$. In vitro invasion of small-cell lung cancer cell lines correlates with expression of epidermal growth factor receptor. Br J Cancer 1998; 78: 631-640.

24 Kawano N, Osawa H, Ito T, Nagashima $\mathrm{Y}$, Hirahara F, Inayama $\mathrm{Y}$ et al. Expression of gelatinase $A$, tissue inhibitor of metalloproteinases-2, matrilysin, and trypsin(ogen) in lung neoplasms: an immunohistochemical study. Hum Pathol 1997; 28: 613-622.

25 Savaraj N, Wei Y, Unate H, Liu PM, Wu CJ, Wangpaichitr M et al. Redox regulation of matrix metalloproteinase gene family in small cell lung cancer cells. Free Radic Res 2005; 39: 373-381.

26 Boukerche H, Aissaoui H, Prevost C, Hirbec H, Das SK, Su ZZ et al. Src kinase activation is mandatory for MDA-9/syntenin-mediated activation of nuclear factor-kappaB. Oncogene 2010; 29: 3054-3066.

27 Lee JW, Juliano R. Mitogenic signal transduction by integrin- and growth factor receptor-mediated pathways. Mol Cells 2004; 17: 188-202.

28 Feldman LE, Shin KC, Natale RB, Todd RF 3rd. Beta 1 integrin expression on human small cell lung cancer cells. Cancer Res 1991; 51: 1065-1070.

29 Hartmann TN, Burger JA, Glodek A, Fujii N, Burger M. CXCR4 chemokine receptor and integrin signaling co-operate in mediating adhesion and chemoresistance in small cell lung cancer (SCLC) cells. Oncogene 2005; 24: 4462-4471.

$($ (1) $(\Theta)$ This work is licensed under a Creative Commons Attribution-NonCommercial-NoDerivs 3.0 Unported License. To view a copy of this license, visit http:// creativecommons.org/licenses/by-nc-nd/3.0/ 